\title{
McArdle disease in a Turkish woman due to an intronic variant of
}

\section{pygm gene}

\author{
Gülden Diniz $^{1 *}$, Canan Çelik², Oğuz Dikbaş ${ }^{3}$, Aslıhan Duman $^{4}$, Ayşe Feyda Nursal $^{5}$
}

\begin{abstract}
Objective: Glycogen storage disease type 5, also known as McArdle disease, is a hereditary disorder with progressive myopathy. It is characterized by onset of exercise intolerance with premature fatigue and painful muscle cramps in childhood or adolescence. Temporary myoglobinuria may occur after exercise due to rhabdomyolysis. Patients may have mild muscle weakness since childhood. Muscular atrophy with fatty replacement may develop in adult life. McArdle disease is a relatively benign disease and rarely severe myoglobinuria can cause acute renal failure as a complication of widespread rhabdomyolysis. This autosomal recessive disease is caused a homozygous mutation of the PYGM gene encoding muscle enzyme myophosphorylase $\mathrm{C}$.

Case: A 36-year-old woman admitted to the Physical Medicine and Rehabilitation polyclinic of Giresun University Hospital with a complaint of gradually increasing progressive weakness and tiredness since 13 years age. CK serum level has been found to in normal range; even after exercise. The findings demonstrated myogenic abnormalities on electromyography, multiple glycogen-containing vacuoles and undetectable muscle myophosphorylase activity on muscle biopsy. The genetic analysis revealed no pathogenic mutations in exon regions of PYGM gene. But in sequencing analysis, the rs71049658, insertion/ deletion variation in intron 17 was determined. Coenzyme Q10 (CoQ10) 30mg/day, vitamin B6 250mg/day and L-carnitine 1 gr/day treatment protocol has been applied and after two months the treatment, clinical improvement has been achieved.

Conclusion: In this case report, an atypical McArdle case with a diagnostic challenge has been presented. We thought that this polymorphism in the myophosphorylase gene may lead to a severe mosaic alteration in mRNA splicing, including exon skipping, activation of cryptic splice-sites, and exon-intron reorganizations.
\end{abstract}

Key words: McArdle Disease, Myopathy, Myophosphorylase, intronic variant

\section{Introduction}

McArdle disease is one of the most common glycogen storage disorders. Although the exact prevalence is not known, it has been estimated to be 1 in 100,000 patients in the United States (1). McArdle disease also known as type $\mathrm{V}$ glycogen storage disease is a hereditary metabolic disorder with progressive myopathy (2). Organs other than muscle does not affected in McArdle disease. The disease is characterized by exercise intolerance with premature fatigue and painful muscle cramps. Rhabdomyolysis characterized by myalgia, muscle swelling and elevated CK levels and myoglobunuria occurs in some patients $(2,3)$. The typical age of onset is late childhood. Neurological evaluation is generally normal between crises.
Deficiency of this enzyme results in insufficient energy flux in skeletal muscle of the patients suffering from McArdle disease $(4,5)$. It is an autosomal recessive disorder results from the mutation of the gene PYGM encoding muscle enzyme myophosphorylase C. More than 100 mutations in PYGM have been associated with this disorder. Human myophosphorylase gene is located on the chromosome 11q13 and make up of 20 exons. The PYGM gene encodes the muscle isoform of glycogen phosphorylase, which catalyzes and regulates the breakdown of glycogen to glucose-1-phosphate during glycogenolysis.

Received 15-05-2019 Accepted 20-06-2019 Available Online 29-06-2019 Published 30-06-2019

1 Izmir Democracy University, Dept of pathology, İzmir, TR

2 Giresun University, Dept of Physical Medicine and Rehabilitation, Giresun, TR

3 Giresun University, Dept of Internal Medicine, Giresun, TR

4 Giresun University, Dept of Pathology, Giresun, TR

5 Giresun University, Dept of Genetics, Giresun, TR

* Corresponding Author: Gülden Diniz E-mail: gulden.diniz@idu.edu.tr Phone: +90 5422431309 
This metabolic pathway is necessary for the generation of ATP during physical activity. As mutation may occur in every exon of PYGM gene, non-mutant, complete PYGM gene sequencing is generally required for function $(1,6)$. Mutational analysis and myophosphorylase activity in muscle assay can be used to confirm diagnosis.

Sometimes permanent muscle weakness may observe in patients. Permanent weakness especially in proximal muscles starts in advanced age (3).

Herein, we present a female patient with McArdle disease with progressive fatigue, tiredness and proximal muscle weakness with distinctive clinical, biochemical, pathological and genetic mutational findings.

\section{Case}

A 36-year-old woman have presented with the complaint of gradually increasing progressive weakness and tiredness since 13 years old. Her history revealed difficulty in running in childhood period. She has been admitted to our outpatient physical medicine and rehabilitation clinic with increased progressive proximal weakness, muscle pain, early fatigue and gait disturbance for last six months. She complains difficulty in walking uphill and climbing stairs. Strength of proximal muscle groups of upper and lower extremities were grade 3/5. Distal muscles were grade $4 / 5$. Gowers' sign and shortening of Achilles tendons were observed. Pseudohypertrophy in cruris muscles and decreased deep tendon reflexes has been found on physical examination. She experienced the second- wind phenomenon. She had been working as a nursery teacher assistant. Neither family history of myopathy nor consanguinity between her father and mother were recorded. Glucose level $90 \mathrm{mg} / \mathrm{dl}$, urea $19 \mathrm{mg} / \mathrm{dl}$, creatinine $0.3 \mathrm{mg} / \mathrm{dl}$, sodium $143 \mathrm{meq} / \mathrm{L}$, potassium $4.4 \mathrm{meq} / 1$, total calcium $9.3 \mathrm{mg} / \mathrm{dl}$ (8.4-10.2), aspartate transaminase $27 \mathrm{U} / 1$ (14-36), alanine transaminase $53 \mathrm{U} / \mathrm{L}$ (9-52), thyroid stimulating hormone $2.65 \mu \mathrm{IU} / \mathrm{ml}(0.465-4.68)$, vitamin B12 302 pg/ml (239-931), 25 hidroxyvitamin D $18.46 \mathrm{ng} / \mathrm{ml}$, creatinine kinase $91 \mathrm{U} / \mathrm{L}$ (26-192), aldolase $2.5 \mathrm{U} / 1$ (0.7-6) were revealed on laboratory evaluation (Table 1).

Urine analysis was normal. Ischemic forearm exercise test was positive. Electrocardiogram was normal sinus rhythm. Echocardiography was not compatible with cardiomyopathy. Electromyographic study showed myogenic patterns in distal muscles. Lumber vertebra MRI scan revealed L5-S1 disc focal protrusion. At the end of laboratory investigation and radiological examination we decided to perform vastus lateralis incisional muscle biopsy.

\section{Pathological Analysis}

Biopsied striated muscle tissue was analyzed with frozen technique and normal pathological preparation process. Size and shape difference, rare nuclear internalizations and vacuoles like muscle injury signs were found in myofibrils on sections (Figure 1).

Mild increase in interstitial connective tissue intensity was detected on Masson's trichrome staining. Glycogen accumulation as irregular globules was observed on PAS staining (Figure 2). A small number of immature fibers were detected on neonatal myosine staining. Repeated control staining revealed a myophosphorylase enzyme defect (Figure 3).

Material accumulation and amyloidosis was not detected on dPAS, Oil Red O and Crystel Violet staining. Myofibrillar and mitochondrial pathology was not detected on Modified trichrome, NDH-TR, SDH and COX staining technique. Type 2/1 ratio and dispersion was normal on enzyme staining.

It was defined to be non-inflammatory myopathy according to histomorphological, histochemical and immunophenotipical findings. It was diagnosed as McArdle disease according to clinicopathological correlation.

Table 1: Laboratory findings of the patient.

\begin{tabular}{|lll|}
\hline Biochemical blood test & Sample value & Standard Range \\
\hline Glucose & $90 \mathrm{mg} / \mathrm{dl}$ & $72-99 \mathrm{mg} / \mathrm{dl}$ \\
\hline Urea & $19 \mathrm{mg} / \mathrm{dl}$ & $7-20 \mathrm{mg} / \mathrm{dl}$ \\
\hline Creatinine & $0.3 \mathrm{mg} / \mathrm{dl}$ & $\mathrm{mg} / \mathrm{dl}$ \\
\hline Total calcium & $9.3 \mathrm{mg} / \mathrm{dl}$ & $8.4-10.2 \mathrm{mg} / \mathrm{dl}$ \\
\hline Aspartate transaminase & $27 \mathrm{U} / \mathrm{l}$ & $14-36 \mathrm{U} / \mathrm{l}$ \\
\hline Alanine transaminase & $53 \mathrm{U} / \mathrm{l}$ & $9-32 \mathrm{U} / 1$ \\
\hline TSH (thyroid stimulating hormone) & $2.65 \mu \mathrm{IU} / \mathrm{ml}$ & $0.465-4.68 \mu \mathrm{IU} / \mathrm{ml}$ \\
\hline Creatinine kinase & $91 \mathrm{U} / \mathrm{l}$ & $26-192 \mathrm{U} / 1$ \\
\hline Vitamin D & $18.46 \mathrm{ng} / \mathrm{ml}$ & $25-80 \mathrm{ng} / \mathrm{ml}$ \\
\hline Vitamin B12 & $302 \mathrm{pg} / \mathrm{ml}$ & $239-931 \mathrm{pg} / \mathrm{ml}$ \\
\hline Sodium & $143 \mathrm{meq} / \mathrm{l}$ & $135-145 \mathrm{meq} / 1$ \\
\hline Potassium & $4.4 \mathrm{meq} / \mathrm{l}$ & $3.5-5.0 \mathrm{meq} / \mathrm{l}$ \\
\hline Aldolase & $2.5 \mathrm{U} / \mathrm{l}$ & $0.7-6 \mathrm{U} / 1$ \\
\hline
\end{tabular}




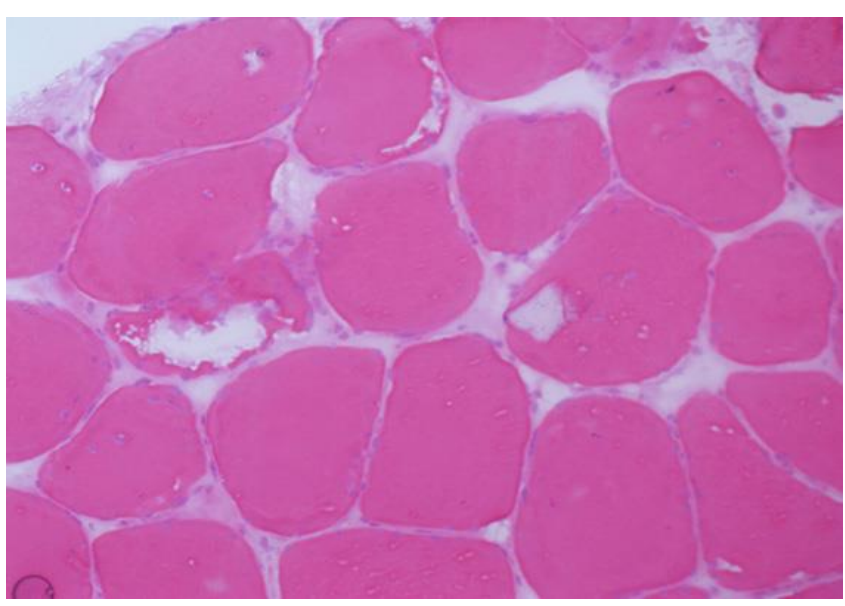

Figure 1: Presence of small vacuoles (HEX 400).

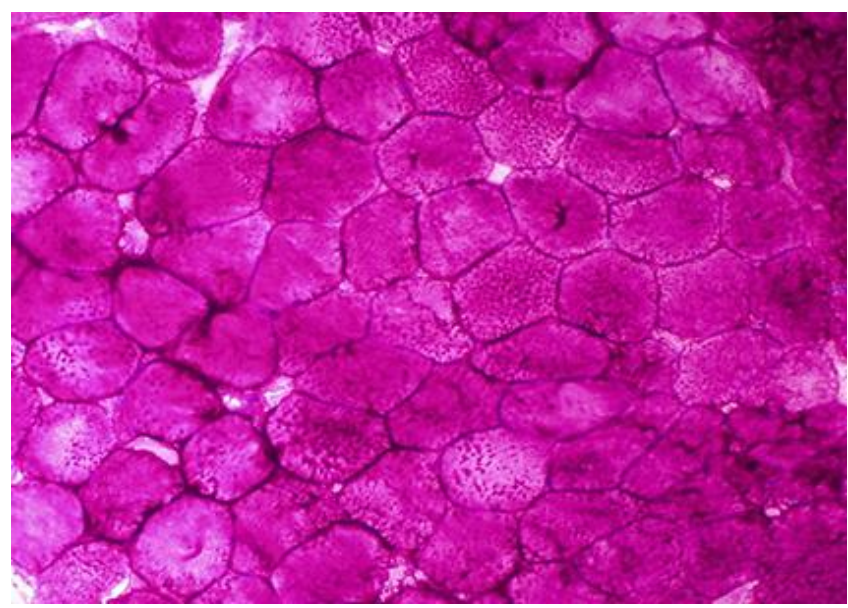

Figure 2: Irregular glycogen accumulation with Periodic Acid Shiff staining (PAS X 200)

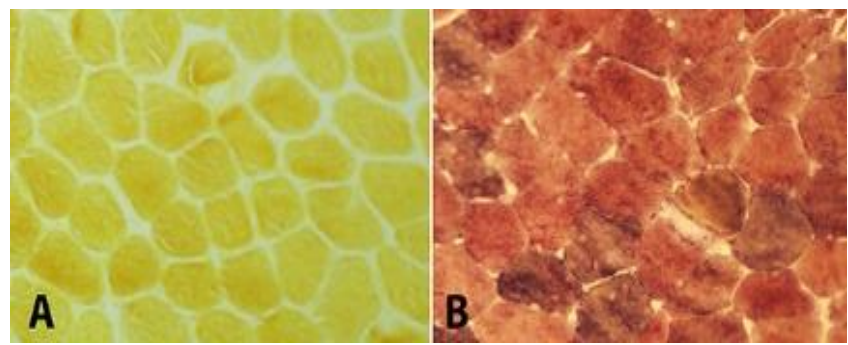

Figure 3: Myophosphorylase enzyme defect in muscle biopsy of this case (A) and control case (B).

\section{Molecular and genetic analysis}

Following to DNA extraction from blood sample, quality control analysis were performed. Initial spectrophotometric measurements were performed with Nanodrop 2000c (Thermo Fisher Scientific, USA). According to test results, the DNA sample was measured as more than $50 \mathrm{ng} / \mu \mathrm{l}$ with 1.8-2.0 for A260/280 analysis. Structural integrity of genomic DNA sample was tested with gel electrophoresis. Genomic sequences of PYGM gene was obtained from UCSC Genome Bioinformatics Database (https://genome.ucsc.edu). Exomes, introns, promoter or exon-intron junctions were targeted for primer design according to genome browser of California University Santa Cruz Genomics Institute. Before to sequencing, the DNA sample was amplified with long range primers that cover exons (plus exon-intron boundary regions with 50 $\mathrm{bp)}$ of PYGM gene. Library preparation was accomplished via a standard PCR conditions for all 3 amplicons at $60^{\circ} \mathrm{C}$ annealing temperature.

Amplified PCR products were prepared by Nextera XT (illumina) protocol. The purification steps in library preparation were performed with Agencourt AMPure XP (Beckman Coulter, Indianapolis, IN, USA) kit. After the library preparation step, amplified and indexed PCR products were sequenced with MiSeq ${ }^{\circledR}$ nextgeneration sequencing platform (Illumine, San Diego, CA, USA).

In analysis pipeline, paired-end raw sequence data was used that obtained from MiSeq ${ }^{\circledR}$ next generation sequencing platform. Sequence data was trimmed from both 3' and 5' end with quality threshold Q10 via Trimmomatic. Sequences were aligned to reference sequences of PYGM Gene (NM 005609.2). After sequence alignment; following analysis steps were performed: local indel realignment (GATK), mark duplicates (Picard), base quality score Recallibration (GATK), variant SNP detection (GATK), variant indel detection (GATK), strand bias filter $<\% 20$ (GATK), and alternative variant read frequency<\%20 (GATK). Filtered variants in the sample are rs555974, rs9704315, rs1207113, rs71049658, rs7126110, rs565688, rs2959652, rs625172, rs630966, rs589691, rs490980, and rs489192 (Table 2).

\section{Results}

Because of pathological glycogen accumulation and myophosphorylase enzyme defect in the muscle biopsy, it was suspected as McArdle disease. The genetic analysis revealed no pathogenic mutations in exon regions of PYGM gene. But in sequencing analysis, an insertion/ deletion variation (rs71049658), which has not been associated with McArdle disease previously, in intron 17 of PYGM gene was determined. Finally, she was diagnosed as McArdle disease according to the genetic findings and clinicopathological correlation.

There is no any standardized treatment for McArdle disease. Creatinine monohydrate, branched chain amino acids, pyridoxine (vitamin B6) has been used for the treatment of this disease. There is no effective gene therapy for McArdle disease yet (7). Dietary precaution with high carbohydrate and low fat like vegetables, fruits, cereals and bread are good as a source of food supplementation. 20-40 gr glucose or fructose before strenuous exercise is better recommended. Regular aerobic exercise is also recommended.

We started Coenzyme Q10 (CoQ-10) 30mg/day, vitamin B6 $250 \mathrm{mg} /$ day and L-carnitene $1 \mathrm{gr} /$ day and after two months treatment, clinical improvement has been detected. She has been recommended moderate aerobic exercise and to avoid heavy static contractions and intense dynamic aerobic exercise. 
Table 2: Filtered variants in the patient sample.

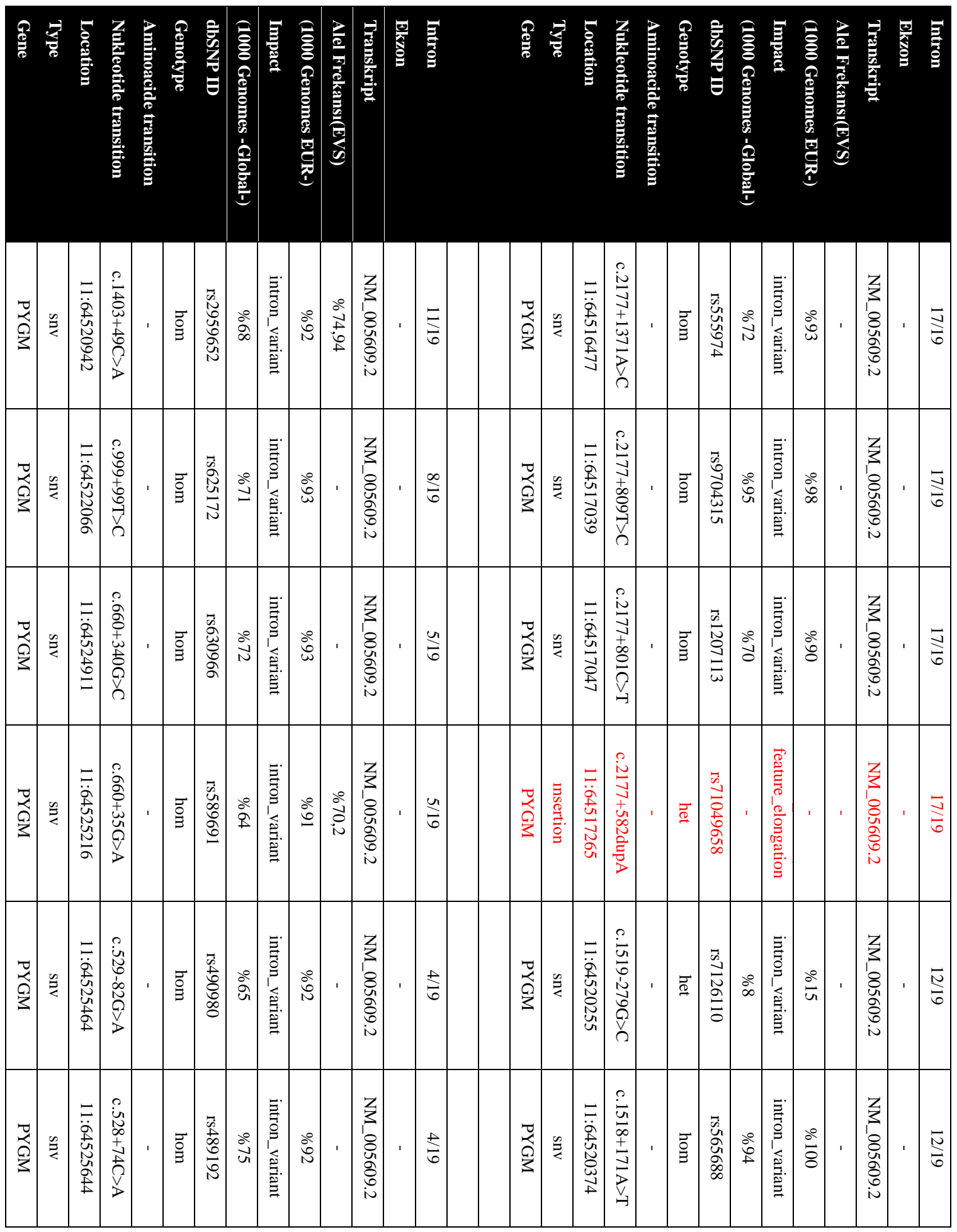




\section{Discussion}

Glycogen storage disease type 5 is a metabolic myopathy with onset frequently in the first decade of life. Clinical heterogeneity exists; some individuals have mild symptoms manifesting as fatigue, whereas a severe, rapidly progressive form manifests shortly after birth. In some individuals, progressive weakness manifests in the sixth or seventh decade of life. The fixed weakness that occurs in approximately one fourth of affected individuals is more likely to involve proximal muscles and is more common in individuals over age 40 years. $(3,4)$. Nadaj-Peklaza et al. reported that proximal fixed muscle weakness was a frequent finding in McArdle disease and 37,5\% of patients were affected after the age of 40 years (3). Permanent muscle weakness has been reported as mild and predominantly effecting proximal other than distal muscles (4). As in the literature, our patient's findings especially proximal weakness had emerged in the late period. Myophsphorylase initiates the breakdown of muscle glycogen by removing (1-4)- $\alpha$-glycosyl unites from the outer branches of glycogen, leading to glycose-1 phosphate that undergoes glycogenolysis. In McArdle disease glycogen stores in muscle could not been used as an energy source. On the other side blood borne glucose converted to glucose- 6 phosphate in the muscle and used as an energy source which explains pre-exercise ingestion of carbohydrate improves exercise tolerance (3).

The four diagnostic tools for McArdle disease are exercise intolerance, high level of serum CK even during rest time, CK elevation following intense exercise, and the second wind phenomena following 10 minutes exercise. The other diagnostic tool is forearm ischemia test which is generally painful procedure. This is a rare case of McArdle disease presenting with symptoms of progressive weakness and tiredness with discordance between pathological and genetic findings and biochemical analysis and tests. Although pathological evaluation of specimen is highly compatible with McArdle disease; there is conflicting findings with clinical and biochemical results. There is not any increase in serum CK level in this patient which is not compatible with myopathy, but clinically there is positive forearm ischemia test observed that is compatible with the diagnosis. Serum CK is elevated in almost all patients with GSD-5, even in the absence of exercise. Serum CK level is rarely normal. Serum CK level at rest did not correlate with clinical severity of the disease (5). CK serum level was found to be normal in our patient; even after exercise.

Glycogen accumulation as irregular globules was observed on PAS staining. A small number of immature fibers were detected on neonatal myosine staining. Repeated control staining revealed a myophosphorylase enzyme defect. Myophosphorylase enzyme defect is highly compatible with McArdle Disease $(1,2)$.

It was defined to be noninflammatory myopathy according to histomorphological, histochemical and immunophenotipical findings. It was primarily thought to be McArdle disease according to clinicopathological correlation. The presence of subsarcolemmal vacuolization is pathognomonic for McArdle disesae which was also detected in our patient (1).

McArdle disease results from different mutations of human myophosphorylase gene (PYGM). PYGM gene encodes the skeletal muscle isoform of glycogen phosphorylase and localized on chromosome 11 (11q12-q13.2). One hundred forty seven pathogenic mutations and 39 polymorphisms in PYMG gene have been reported until 2015. Exon 1 and 17 are mutational hot-spots in PYGM and 50\% of the described mutations are missense. Only 9.5\% of PYMG gene mutations are intronic variations (6). The majority of intronic polymorphisms are found in introns 5, 16, and 17, whereas the rest are equally distributed throughout the remaining introns. In a study, apart from the 39 polymorphisms found in the PYGM gene, an additional list of 200 polymorphisms were cited for the PYGM gene. Nogales Gadia, et al. listed the molecular findings in a Spanish patient with McArdle disease who harbored a silent polymorphism [c.1827G >A (p.K609K)] in the myophosphorylase gene (6). They reported that, this apparently silent PYGM polymorphism was actually found to severely alter mRNA splicing, and as such was finally classified as a pathogenic mutation instead of a polymorphism.

\section{Conclusion}

Here we present a case with a diagnostic challenge. This is an atypical McArdle disease with clinicopathologic discordance. PYGM, MIM \#608455 gene sequencing analysis was planned after the pathological results. In sequencing analysis, it was determined a variation in PYGM gene which has not been associated with McArdle disease previously (rs71049658), insertion/ deletion variation in intron 17. That is new finding for McArdle disease $(1,6)$. We thought that this polymorphism may lead to some alterations in mRNA splicing, including exon skipping, activation of cryptic splice-sites, and exon- intron reorganizations.

Therefore it may suggest that, in patients with McArdle disease in whom no pathogenic mutation has been found in exons, any silent polymorphism should be re-evaluated as a putative splicing mutation. This reported polymorphism in our patient can be responsible the deterioration in myophosphorylase function. When genome variants are identified in genomic DNA, especially during routine analysis of disease- associated genes, their functional implications might not be immediately evident.

Distinguishing between a genomic variant that changes the phenotype and one that does not is a difficult task. An increasing amount of evidence indicates that genomic variants in both coding and non-coding sequences can have unexpected deleterious effects on the splicing of the gene transcript. Only with further researches, benign polymorphisms be distinguished from disease- associated splicing mutations.

Acknowledgement: We gratefully thank to firm "DONE Genetics and Bioinformatic" for their support in genetic analysis of this case. 
Conflict of Interest: The authors declare no potential conflicts of interest with respect to the research, authorship, and/or publication of this article.

Author's Contributions: GD, CÇ, OD, AD; Research concept and design, Patient examination, Research the literature, preparation of the article. AFN; Genetic Analsis. GD; Revision of the article.

\section{References}

1. De Castro M, Johnston J, Biesecker L. Determining the prevalence of Mc Ardle disease from gene frequency by analysis of nextgeneration sequencing data. Genet Med 2015;17(12):1002-6. doi: 10.1038/gim.2015.9

2. Vissing J, Duno M, Schwartz M, Haller RG. Splice mutations preserve myophosphorylase activity that ameliorates the phenotype in Mc Ardle disease. Brain 2009; 132 (6): 1545-1552.
3. Nadaj-Pakleza AA, Vincitorio CM, Laforet P, Eymard B, Dion E, Teijeira $\mathrm{S}$, et al. Permanent muscle weakness in McArdle disease. Muscle \& nerve 2009; 40(3):350-7.

4. Satoh A, Hirashio S, Arima T, Yamada Y, Irifuku T, Ishibashi H, et al. Novel Asp511Thr mutation in McArdle disease with acute kidney injury caused by rhabdomyolysis. CEN Case Rep 2019 Mar 21. doi: 10.1007/s13730-019-00392-6

5. Scalco RS, Morrow JM, Booth S, Chatfield S, Godfrey R, Quinlivan R. Misdiagnosis is an important factor for diagnostic delay in McArdle disease. Neuromuscul Disord 2017;27(9):852-855. doi: 10.1016/j.nmd.2017.04.013.

6. Nogales-Gadea G, Brull A, Santalla A, Andreu AL, Arenas J, Martin MA, et al. McArdle Disease: Update of Reported Mutations and Polymorphisms in the PYGM Gene. Human mutation 2015; 36(7):669-78.

7. Quinlivan R, Martinuzzi A, Schoser B. Pharmacological and nutritional treatment for McArdle disease (Glycogen Storage Disease type V). The Cochrane database of systematic reviews 2014;11:CD003458. 